\title{
A Review on Carbon Nanotubes: As a Nano carrier Drug Delivery System
}

\author{
S. PALIWAL* ${ }^{*}$ K. PANDEY, S. PAWAR, H. JOSHI AND N. BISHT \\ College of Pharmacy, Graphic Era Hill University, Bhimtal Campus, Sattal Road Nainital-263156, Uttarakhand, India
}

Paliwal et al.: Review on Carbon Nanotubes

\begin{abstract}
Carbon nanotubes were discovered in 1991, Sumio Iijima gives the theory of carbon nanotubes which is made up of carbon allotrope graphene having brilliant electrical, mechanical, optical and synthetic properties, pulling in extensive enthusiasm for their application in different fields including drug conveyance and malignancy treatment. Malignancy is one of the most testing sicknesses of present day times since its treatment includes recognizing ordinary sound cells from influenced cells. Here, carbon nanotubes assume a significant job since marvels, for example, electronic paramagnetic resonance, permit carbon nanotubes to recognize typical cells from influenced ones, the Holy Grail in malignancy treatment. Significant work has been done on carbon nanotubes as medication conveyance frameworks throughout the most recent 20 y. Not with standing, worries over specific issues, for example, biocompatibility and poisonousness have been raised and warrant broad exploration in this field. For the preparation of Single-walled carbon nanotubes and Multi-walled carbon nanotubes arc discharge, laser ablation and chemical vapor deposition method of preparation are used. By the process of acid treatment, magnetic purification and size exclusion chromatography carbon nanotubes impurities are removed out. Because of hydrophobic nature of carbon nanotubes, Covalent and non-covalent functionalization are done for the surface modification. Cytotoxic of carbon nanotubes have a beneficial effect in cancer treatment. Drug released from carbon nanotubes by using various stimulants such as electric, magnetic field and changes in $\mathrm{pH}$.
\end{abstract}

Key words: Carbon nanotubes /use/drugs delivery, SWCNT, MWCNT, graphene, functionalization, cytotoxicity

In Carbon nanotubes (CNTs) are the tubes which made up of carbon allotrope graphene which has less than $100 \mathrm{~nm}$ diameter. CNTs are discovered by Sumio Iijima in 1991, at that time the spherical carbon molecules are known as fullerenes. Basically, these are the allotropes of carbon with a cylindrical nanostructure and are composed of a graphene sheet with $\mathrm{sp}^{2}$-hybridized carbon atoms. The connection of tightly bond of $\mathrm{sp}^{2}-$ hybridized carbon atoms provide the CNTs mechanical strength and the special rolling pattern of graphene sheets at different chiral centers contributes to their physicochemical and electrical properties, they have a higher tensile strength than steel and Kevlar. This bond is even stronger than the $\mathrm{sp}^{3}$ bond found in diamond. Single-walled carbon nanotubes (SWCNTs) have hundreds of times more tensile strength than steel ${ }^{[1,2]}$.

\section{PROPERTIES OF CARBON NANOTUBES}

It has high thermal conductivity. Carbon Nanotubes have high electrical conductivity and low thermal expansion coefficient. Carbon Nanotubes are elastic in

*Address for correspondence

E-mail: soniapaliwal49@gmail.com

September-October 2020 nature $18 \%$ elongations to failure. Carbon Nanotubes have very high tensile strength and highly flexible (i.e.) they can be bent without damage.

The structure of graphene in carbon atoms (a single sheet of graphite) forms a planar honeycomb lattice and each atom is bind to three neighboring atoms by a strong chemical bond. CNTs represent a very small, high-aspect-ratio conductive additive for all kinds of plastics where the high aspect ratio means that a lower loading (concentration) of CNTs is required to realize the same electrical conductivity when compared to other conductive additives. This low loading preserves the polymer resins toughness, especially at low temperatures. In a maximum proportion carbon This is an open access article distributed under the terms of the Creative
Commons Attribution-NonCommercial-ShareAlike 3.0 License, which
allows others to remix, tweak, and build upon the work non-commercially,
as long as the author is credited and the new creations are licensed under
the identical terms

Accepted 14 October 2020

Revised 09 October 2020

Received 11 May 2020

Indian J Pharm Sci 2020;82(5):766-772 
is present in human body which is a considered as a biocompatible material. The cells growth on CNTs has been demonstrated; therefore, they apparently have no toxic effect. The cells also do not adhere to the CNTs, opening doors for applications such as anti-fouling coatings for ships and coatings for prosthetics. The wall of CNTs is of two types: Single-walled CNTs formed by rolling up of single sheet graphene to form a hollow tube (SWCNTs). Multi-walled CNTs (MWCNTs) are formed by rolling the multiple concentric layers of graphene composing it.

Both CNTs are enclosed at both ends in a hemispherical arrangement of carbon networks called fullerenes covered up by the graphene sheet. CNTs may have different lengths ranging from several hundred nano meters to several millimetres, but their diameters depend on their class. As SWCNTs are 0.4-3 nm in diameter and MWCNTs are 2-500 nm in diameter, depending on the method of synthesis ${ }^{[3]}$. A Singlewalled carbon nanotube are composed of a single layer of graphene which forms a cylindrical shape, which can be observed as two planes under a transmission electron microscope. Whereas, a Multi-walled carbon nanotube is made of multiple layers of graphene, which is formed in a concentric pattern around the smallest nanotube ${ }^{[4]}$.

The gap between the graphene interlayer of MWCNTs measures approximately $0.34 \mathrm{~nm}$ on average, each forming an individual tube, with all the tubes having a larger outer diameter $(2.5-100 \mathrm{~nm})$ than SWCNTs (0.6$2.4 \mathrm{~nm}$ ).SWCNTs have a better defined wall, whereas MWCNTs are more likely to have structural defects, resulting in a less stable nanostructure ${ }^{[5]}$.

\section{METHOD OF PREPARATION}

\section{Arc discharge method:}

The chamber is composed of two vertical graphite rods one of which is cathode while other is an anode evaporated carbon molecule, and some metal catalysts such as nickel, cobalt, and/or iron (fig. 1).
The direct current is passed through the chamber, which is called an arcing process, and the chamber is being pressurized as well as heated to approximately $4000^{\circ}$. During the process, about half of the evaporated carbon solidifies on the cathode (negative electrode) tip, at a rate of $1 \mathrm{~mm} / \mathrm{min}$, whereas the anode (positive electrode) is consumed ${ }^{[6]}$. In the arc discharge synthesis of CNTs, there are two main methods: Synthesis with the use of different catalyst precursors. Without using any catalyst precursor

Generally, synthesis of multi-walled carbon nanotubes (MWCNTs) could be done without using catalyst precursors, whereas synthesis of single-wall carbon nanotubes (SWCNTs) utilizes different catalyst precursors and, for expansion in arc discharge, utilizes a complex anode, which is made from a composition of graphite and a metal ${ }^{[7]}$.

Advantage: The arc discharge technique produces a large quantity of nanotubes

Disadvantage: The process requires high temperature for CNT synthesis, resulting in fewer structural defects (due to expansion of CTNs).

\section{Laser ablation Method:}

A graphite target is vaporized by laser in an inert atmosphere under high temperature in this method, at the same time the laser produces carbon species, which were transported to a conical, water-cooled copper collector by the flowing inert gas from the hightemperature zone ${ }^{[8]}$ (fig. 2).

When the transition metal such as $\mathrm{Ni}, \mathrm{Co}$, or $\mathrm{Fe}$ is added in a small quantity to the carbon target the Single Walled Carbon nanotubes were produced. The yield of CTNs by this method is totally temperature dependent. By varying the laser, growth temperature, catalyst composition, nature of gases, and gas pressure, the average nanotube diameter and size distribution can be $\operatorname{varied}^{[9]}$.

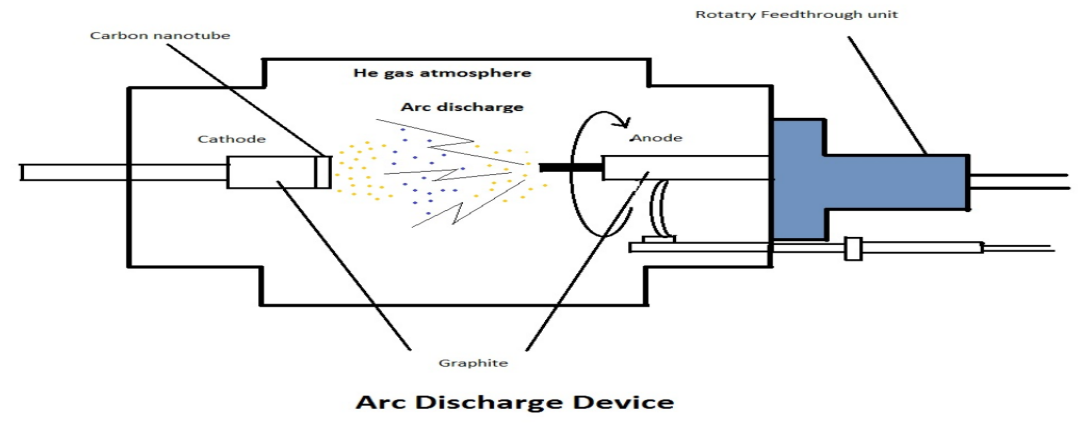

Fig. 1 Arc discharge device 
Advantage: High yield with relatively low metallic impurities

Disadvantage: CNTs prepared by laser ablation method are not uniformly straight but having some branching.

\section{Chemical vapor deposition:}

Chemical vapor deposition (CVD) is the method for large-scale production of CTNs. By this method, the decomposition of the carbon precursor and CNT are obtained on the surface of catalyst particles (fig. 3).

The two most important CVD techniques for synthesis of CNTs are as follows: the thermal $\mathrm{CVD}^{[10]}$. Plasmaenhanced CVD

In this method a carbon source such as methane, carbon monoxide, acetylene, etc is used as an energy source basically in the gas phase. CNT synthesis using CVD is basically a two-step process. At first the catalyst is prepared by physical vapor deposition or sputtering or dip coating, etc. Then the substrate is heated in a carbonrich gaseous environment in 500-1000 ${ }^{\circ 11]}$. Comparison between all methods depicted in Table 1 .

Advantage: Economically compatible method, resulting in large scale production of pure $\mathrm{CTNs}^{[12]}$.

Disadvantages: It uses large scale synthesis routes.

\section{PURIFICATION OF CNTS}

Metallic impurities were found to be present in Carbon Nanotubes which are able to create various type of toxicity, becoming a major issue with CTNs based on various researches. The major contaminants comprise metals such as iron, cobalt, nickel etc. Which basically get introduced during manufacturing process of CTNs leading to loss in efficiency of CTNs.

\section{Acid treatment:}

To remove the metal catalyst from CNTs the acid treatment can be opted which includes the following steps: The surface of the metal is exposed to oxidation or sonication. Followed by the exposure of the metal catalyst is to acid and solvated. For which Nitric acid generally as it has effect only on the metal catalyst ${ }^{[14]}$.

\section{Magnetic purification:}

In this method the ferromagnetic particles are mechanically removed from their graphitic shells. The catalytic particles are removed.

\section{Size exclusion chromatography:}

For separating semiconducting and metallic SWCNTs size exclusion chromatography of DNA-dispersed

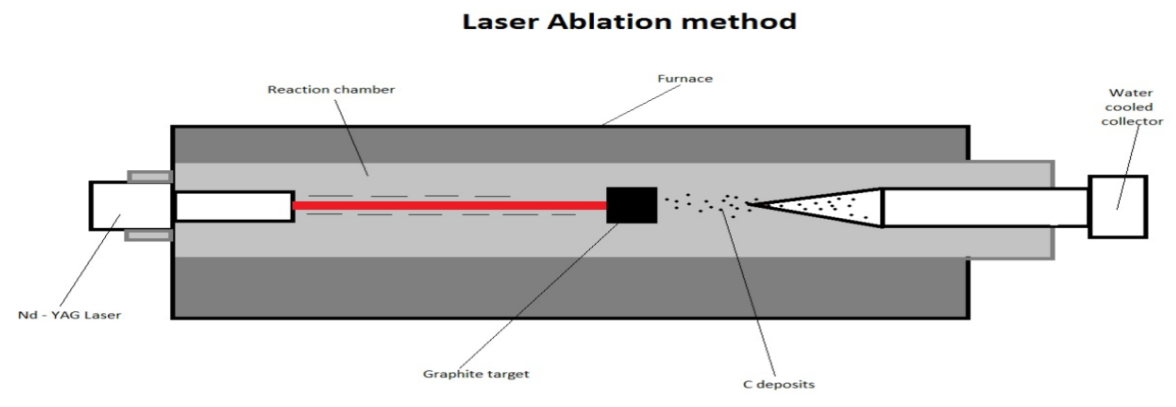

Fig. 2 Laser ablation method

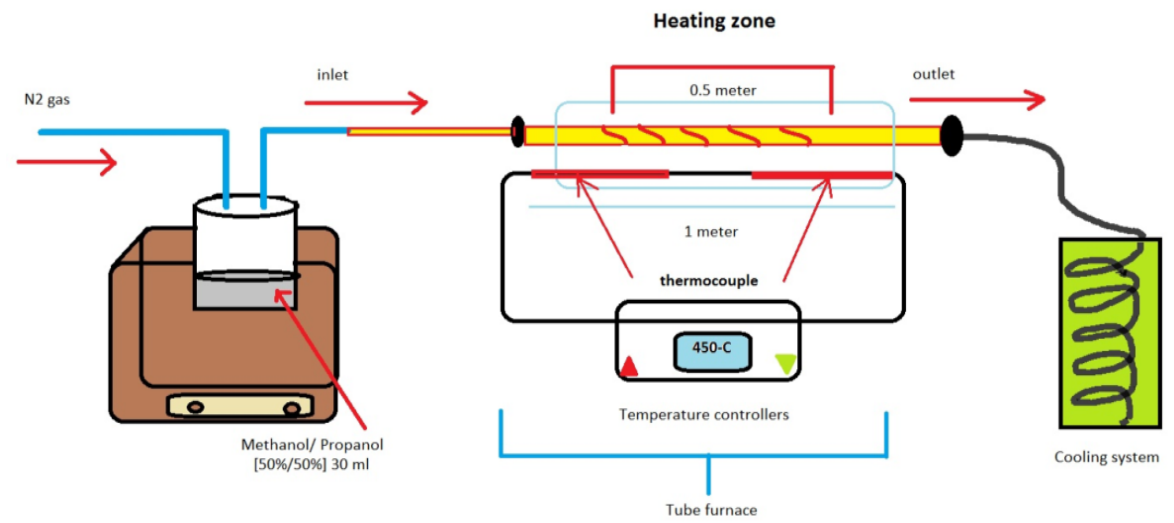

Fig. 3 Chemical vapor deposition method 
www.ijpsonline.com

TABLE 1: COMPARISION BETWEEN METHODS OF PREPARATION OF CNT ${ }^{[13]}$

\begin{tabular}{|c|c|c|c|}
\hline Method & Arc discharge & Laser ablation & Chemical vapor deposition \\
\hline Condition & Inert Gas like Helium, Argon & $\begin{array}{l}\text { High laser beam at high temp 500- } \\
1000^{\circ} .\end{array}$ & $\begin{array}{l}\text { Low pressure inert gas like } \\
\text { Nitrogen, Argon }\end{array}$ \\
\hline Yield & Low (20-100 \%) & Average (up to $70 \%$ ) & High (70-90 \%) \\
\hline Purity & Medium & Low & High \\
\hline Temperature & $\sim 4000^{\circ}$ & $25-1000^{\circ}$ & $500-1000^{\circ}$ \\
\hline \multirow[b]{2}{*}{ Product } & SWCNTs: diameters of $0.6-1.4 \mathrm{~nm}$ & $\begin{array}{l}\text { SWCNTs: long bundles of tubes (5- } \\
20 \mu \mathrm{m} \text { ) with individual diameter from } \\
1 \text { to } 2 \mathrm{~nm}\end{array}$ & $\begin{array}{l}\text { SWCNTs: long tubes with } \\
\text { diameters ranging from } 0.6 \\
\text { to } 4 \mathrm{~nm}\end{array}$ \\
\hline & $\begin{array}{l}\text { MWCNTs: short tubes } \\
\text { with inner diameter of } \\
1-3 \mathrm{~nm} \text { and outer diameter of } \\
\text { approximately } 10 \mathrm{~nm}\end{array}$ & MWCNTs: not useful & $\begin{array}{l}\text { MWCNTs: long tubes with } \\
\text { diameter ranging from } 10 \text { to } \\
240 \mathrm{~nm}\end{array}$ \\
\hline
\end{tabular}

Carbon nanotubes is used. This is one of the HPLC separation models which have the highest resolution length sorting ${ }^{[15]}$.

\section{FUNCTIONALIZATION OF CARBON NANOTUBES}

There are some demerits in Conventional Carbon Nanotubes such as poor water solubility because of their hydrophobic nature, however this property can be conveniently modified by surface functionalization (The functionalization of the CNT surface is a promising means of overcoming these problems by the attachment of organic or inorganic moieties to their tubular structure). Physical properties such as wettability and decreasing the interbond attraction are key factors in enhancing the aqueous solubility of CNTs. The functionalized CNTs present enhanced properties enabling facile production of novel nanomaterials and Nano devices. The functionalization of CNTs could get better their chemical compatibility and dissolution properties, which would enable both a wider characterization and resultant chemical reactivity. Apart from water solubility, other factors such as drugcarrying capacity, cellular uptake, absorption profile and excretion from biological system can also modified by the process of functionalization ${ }^{[16]}$. Treatment of CNTs with strong acids results in the introduction of carboxyl groups on their surfaces which increases their dispersibility in aqueous solutions. CNTs are currently one of the most popular nanomaterials used in a number of applications including electronics, energy storage, solar cells, molecular separation, sensing, bio sensing and drug delivery gene therapy etc. CNTs can be used as drug-delivery vehicles or 'Nano carriers' in cancer therapy and other areas of medicine without causing toxicity in healthy tissue and allowing prolonged drug release. In current scenario carbon nanotubes (CNTs) are resourceful subject of medical, effective drug regimen and in the bio sensing methods for treatment and health monitoring especially in cancer therapy primarily includes surgery, radiation therapy, and chemotherapy ${ }^{[17]}$.

To act as a good drug delivery system the solubility and biocompatibility are the basic necessities, hence the first property CNTs must possess is that they be easily soluble in the gastrointestinal environment which require aqueous solubility along with easy and uniform dispersion. The basic stumbling block in the path of better drug delivery practically is solubility of CNTs in aqueous medium due to graphene side walls having $\pi-\pi$ interactions. For obtaining better solubility and dispersion the process of functionalization is one of the effective approaches which enhance the wetting of the hydrophobic tube surfaces and decreasing the CTNs bundle formation. To obtain desirable dispersion, Foldvari et al. have proposed four basic approaches ${ }^{[18]}$ : surfactant-assisted dispersion, solvent dispersion, functionalization of side walls, and bimolecular dispersion. With the help of functionalization unwanted properties such as cytotoxicity etc. can be reduced, alteration in the physiochemical properties can be done $^{[19]}$. The functionalization can be divided into two main subcategories: non-covalent functionalization and covalent functionalization. Basically, functionalization can be done in two ways, i.e., covalent functionalization and non-covalent functionalization ${ }^{[20]}$. Even entrapment of molecules in the internal cavity of CNTs is also done via functionalization. Sites for Functionalization in CNTs are The side wall of CNTs. The end cap of CNTs.

\section{Covalent functionalization:}

Covalent functionalization gives the more secure conjunction of functional molecules. Cycloaddition and oxidation are two most widely employed 
techniques for covalent functionalization of CNTs. The covalent functionalization of graphene can be achieved by nucleophilic substitution, electrophilic addition, and addition and condensation reactions. The mechanism involves in the covalent functionalization is to establishment of covalent linkage between the unsaturated carbon $\pi$-bond and other functional groups. Oxidation of CNTs can be brought about by reacting them with strong acids such as $\mathrm{HNO}_{3}, \mathrm{H}_{2} \mathrm{SO}_{4} / \mathrm{HNO}_{3}$, or $\mathrm{KMnO}_{4} / \mathrm{H}_{2} \mathrm{SO}_{4}$. Upon oxidation, a carboxylic functional group $(\mathrm{COOH})$ is introduced to the $\mathrm{CNTs}^{[21]}$. Functionalization with $\mathrm{COOH}$ provides a link for ligation of other molecules onto the CNTs so as to achieve any required effects. These linkages may be further worked out through amidation or esterification reactions that are suitable for the attachment of molecules without inducing any alteration in the electronic properties of CNTs. Some disadvantages of oxidation reactions are shortening of CNTs and the development of defects on open end. To overcome these problem functional groups on the oxidized CNTs can further react with SOC land carbodiimide to yield functional materials with great propensity for reacting with other compounds ${ }^{[22]}$. Cycloaddition is sometimes a better alternative to overcome the above-mentioned problems. 1,3-Dipolar cycloaddition reaction is mostly adopted for better functionalization based on the fact that upon reaction, amino acids and aldehydes condense, leading to the formation of azomethine ylide adduct, which in turn reacts with the side wall of CNTs. This results in the formation of a pyrrolidine ring which facilitates conjugation of drug molecules ${ }^{[23]}$.

\section{Non Covalent functionalization:}

This method does not affect the chemical properties of CNTs it is totally dependent on $\pi \pi$ interaction, vanderwals forces, and/or hydrogen bonding. It is found that this method is quite easier than the covalent functionalization. Functionalization of proteins, singlestranded DNA and also fluorescence-attached PEG are some of the examples of non-covalent functionalization achieved through $\pi-\pi$ interaction $^{[24]}$.

\section{DRUG LOADING}

As CNTs is the form of targeted drug delivery system, which deliver the drug to the desired site. So, loading of the drug means the active form of drug is inserted into the Drug delivery system which is in the form of CNTs combined with the other carriers. CNTs are having high surface area and volume because of which they can carry drug in a large amount. Its hydrophilic nature can be further improved by functionalization of CNTs.

SW/MWCNTs (3 mg) were redispersed in $15 \mathrm{ml}$ of deionized water by probe sonication. Drug was dissolved in $1 \mathrm{ml}$ of solvent and added drop wise to the SW/MWCNTs solution under vigorous stirring and incubated overnight stirring at room temperature. The resultant drug loaded SW/MWCNTs were centrifuged at $10000 \mathrm{rpm}$ for $15 \mathrm{~min}$ to remove the free drug. The amount of free drug in the supernatant was determined by measuring the absorbance at $440 \mathrm{~nm}$ to estimate its concentration from the dose absorption curve, allowing the drug loading efficiency to be estimated. The drug loading efficiency (DLE, wt \%) of drug in SW/ MWCNTs was calculated according to the following formula:

$D L E \%=$ weight of CUR added-weight of free CUR in supernatant/ weight of CUR added $\times 100$

\section{CYTOTOXICITY OF CNTS}

Toxic effect of CNTs is no doubts a hot topic rising with the study of nanotechnology. Study of cytotoxicity of CNTs is completely different from other toxicological studies. To deal with cytotoxicity of CNTs one must have proper knowledge about the nature and behavior of CNTs. Some researcher report showed the cytotoxicity of CNTs to mammalian cells whereas others expounded their biocompatibility. Chen applied a specific strategy to evaluate cytotoxicity associated with CNTs. It involved biomarkers that measures changes in gene expression (due to initiation of inflammation, apoptosis or immune response) on exposure to nanomaterials. This cytotoxic behavior of CNTs could be used in a positive manner for treatment of cancer ${ }^{[25]}$. Some other properties such as surface area, dispersal properties, functionalization and biocompatibility of CNTs may also be responsible for creating cytotoxicity in mammals. Some studies have also showed that with the help of functionalization the biocompatibility of CNTs can be improved and level of toxicity can be reduced as compared to those with nonfunctionalized CNTs ${ }^{[26]}$. Different researches have been made to reduce the immunotoxicity such as suppressing the size of CTNs may reduce the immunotoxicity. Some other factors can also influence the cytotoxicity such as reduction in recommended dose allowance, coating with biocompatible polymers such as polyurethane, polycarbonate etc ${ }^{[27,28]}$. It was also found that some of the impurities such as Fe causes inhibitory effects in CTNs which in further researches can be removed by various methods such as oxidation (protecting metal 
catalyst) of nanotubes followed by sulfidation method (results in removing carbonaceous impurities present on the surface of nanotubes ${ }^{[29,30]}$. Several researchers have found the degradation of nanotubes with reactive radical intermediates, Alllen et al.in his research found that the functionalized nanotubes were having better oxidizing susceptibility ${ }^{[31]}$. It can be concluded from such studies that functionalization of CNTs may result in increased bioavailability and enhancing its biodegradability.

\section{DRUG RELEASE MECHANISM}

After the preparation and functionalization of CNT's, drug is loaded into the CNT's. So it is important to understand the mechanism to drug release from encapsulated CNT's, to show their therapeutic response. To trigger the drug release from $\mathrm{CNT}$ various stimulants are used such as electric field, magnetic field, changes in $\mathrm{pH}$ around them ${ }^{[32]}$. Ji SI synthesized TDDS of ketoprofen MWCNT's with polyethylene oxide (PEO) and pentaerythritol triacrylate (PETA) polymers and drug release was accelerated by applying a high electric voltage, dissolved the PEO and drug is released from $\mathrm{CNT}^{\text {' }}{ }^{[33]}$. In another study, gemcitabine loaded hyaluronic acid MWCNT's was conjugated with PEGylated using different $\mathrm{pH}$ as a stimulant for drug release and examined the sample by HPLC method ${ }^{[34]}$.

The thermal conductivity and exceptional mechanical properties of CNTs are particularly important for handy applications. Various obstacles still can't seem to be defeated before the handy capability of nanotubes can be acknowledged, not least discovering better and less expensive combination and filtration procedures, improving our capacity to control them at the nanoscale, and the advancement of more delicate portrayal strategies. However, given that their properties are so abnormal, their size is so little and the extension for applications is so enormous, we feel sure that it is just an issue of time before the guarantee of nanotubes is satisfied. In the field of CNT innovation for malignant growth treatment, the issues encompassing CNT harmfulness stay uncertain, as there are various clashing investigations showing both poisonous and non-harmful conduct, notwithstanding various medications which have been conveyed utilizing carbon nanotubes. This is partially; it appears, because of the idea of the exploration being led. That is, there is no genuine benchmark for looking at results. Because of the scope of boundaries recorded before which have been appeared to influence CNTs harmfulness, this is a territory which will require the consistent consideration of toxicologists later on. It is an incredibly encouraging use of nano-innovation and is certainly worth further exploration, as the current strategies for malignant growth treatment are unpredictably hurtful and just somewhat powerful.

\section{Acknowledgement}

The authors thankful to Head of department, College of Pharmacy, Graphic Era Hill University, for providing the necessary facilities and support to carry out the research work.

\section{Conflict of interests:}

The authors declared no conflict of interest.

\section{REFERENCES}

1. Yamaguchi T, Bandow S, Iijima S. Synthesis of carbon nanohorn particles by simple pulsed arc discharge. Che m Phys Lett 2004;389:181-5.

2. Dresselhaus MS, Dresselhaus G, Charlier JC, Hernandez E. Electronic, Thermal and mechanical properties of carbon nanotubes. Philos Transact A Math Phys Eng Sci 2004;362:2065-98.

3. Kim SN, Rusling JF, Papadimitrakopoulos F. Carbon nanotubes for electronic and electrochemical detection of biomolecules. Adv Mater 2007;19:3214-28.

4. Kumar S, Singh Kushwaha, Ghoshal S, Rai AK, Singh S, Singh P. Carbon nanotubes as a novel drug delivery system for anticancer therapy. Braz J Pharm Sci 2013;49:629-43.

5. Kafa H, Wang, JT, Al-Jamal. Current perspective of carbon nanotubes application in neurology. Int Rev Neurobiol 2016;130:229-63.

6. Kaur J, Gill SG, Jeet K. Applications of Carbon Nanotubes in Drug Delivery. Characterization and Biology of Nanomaterials for Drug Delivery 2019;1:113-35.

7. Eatemadi A, Daraee H, Karimkhanloo H, Kouhi M, Zarghami $\mathrm{N}$, Akbarzadeh A, et al. Carbon nanotubes: properties, synthesis, purification, and medical applications. Nanoscale Res Lett 2014;9:393.

8. Thess A, Lee R, Nikolaev P, Dai H, Petit P, Robert J, et al. Crystalline ropes of metallic carbon nanotubes. Science 1996;273(5274):483-7.

9. Yudasaka M, Yamada R, Sensui N, Wilkins T, Ichihashi T, Iijima S. Mechanism of the effect of NiCo, $\mathrm{N}$ and Co catalyst $\mathrm{s}$ on the yield of single-wall carbon nanotubes formed by pulsed Nd:YAG laser ablation. J Phys Chem A 1999;130(30):6224-9.

10. Vander Wal RL, Berger GM, Ticich TM. Carbon nanotube synthesis in a flame using laser ablation for in situ catalyst generation. Appl Phys 2003;77(7):885-9.

11. Purohit R, Purohit K, Rana S, Rana RS, Patel V. Carbon nanotubes and their growth methods. Procedia Mater Sci 2014;6:716-28.

12. Patole SP, Alegaonkar PS, Lee HC, Yoo JB. Optimization of water assisted chemical vapor deposition parameters for super growth of carbon nanotubes. Carbon 2008;46(14):1987-93.

13. Xiao-Di wang, Vinodgopal K, Gui-ping dai. Synthesis of carbon nanotubes by catalytic chemical vapor deposition. intechopen limited. London: UK; 2019;1-19.

14. Rummeli MH, Borowiak-Palen E, Gemming T, Pichler T, 
Knupfer M, Kalbac M, et al. Novel catalysts, room temperature, and the importance of oxygen for the synthesis of single-walled carbon nanotubes. Nano Lett 2005;5(7):1209-15.

15. Huang X, Mclean RS, Zheng M. High-resolution length sorting and purification of DNA-wrapped carbon nanotubes by sizeexclusion chromatography. Anal Chem 2005;77(19):6225-8.

16. Chowdhury DF. Carbon nanotube for drug delivery and controlled release. Biotechnology 2011;5(2):643-55.

17. Tasis D, Tagmatarchis N, Bianco A, Prato M. Chemistry of carbon nanotubes. Chem Rev 2006;106:1105-36.

18. Foldvari M, Bagonluri M. Carbon nanotubes as functional excipients for nanomedicines. Pharmaceutical properties. Nanomedicine 2008;3:173-82.

19. Foldvari M, Bagonluri M. Carbon nanotubes as functional excipients for nanomedicines: II. Drug delivery and biocompatibility issues. Nanomedicine 2008;3:183-200.

20. Chourasia MK, Chaurasia M, Jain NK, Novel carriers for drug delivery. Pharma Med Press 2016;111-43.

21. Prato M, Kostarelos K, Bianco A. Functionalized carbon nanotubes in drug design and discovery. Acc Chem Res 2008;41:60-8.

22. Jain AK, Dubey V, Mehra NK, Lodhi N, Nahar M, Mishra DK, et al. Carbohydrate-conjugated multiwalled carbon nanotubes: development and characterization. Nanomedicine 2009;4:43242.

23. Kafa H, Wang, JT, Al-Jamal. Current perspective of carbon nanotubes application in neurology. Int Rev Neurobiol 2016;130:229-63.

24. Liu Z, Fan AC, Rakhra K, Sherlock S, Goodwin A, Chen X. Supramolecular stacking of doxorubicin on carbon nanotubes for in vivo cancer therapy. Angew Chem Int Ed 2009;48:766872 .
25. Chen F. Toxicology and Cellular Effect of Manufactured Nanomaterials. 2012;35:261-5.

26. Cui HF, Vashist SK, Al-Rubeaan OK, Luong JH, Sheu FS, Interfacing carbon nanotubes with living mammalian cells and cytotoxicity issues. Chem Res Toxicol 2010;23:1131-47.

27. Ursini CL, Cavallo D, Fresegna AM, Ciervo A, Maiello R, Buresti $\mathrm{G}$, et al. Comparative cyto-genotoxicity assessment of functionalized and pristine multiwalled carbon nanotubes on human lung epithelial cells. Toxicol In vitro 2012;26:831-40.

28. Kumara R, Dhanawatb M, Kumara S, Brahma N. Singh C , Jayant K, et al. Carbon Nanotubes: A potential concept for drug delivery applications. Recent Pat Drug Deliv Formul 2014;8:12-26.

29. Meng L, Jiang A, Chen R, Li CZ, Wang L, Qu Y, et al. Inhibitory effects of multiwall carbon nanotubes with high iron impurity on viability and neuronal differentiation in cultured PC12 cells. Toxicol 2013;313:49-58.

30. Goto H, Furuta T, Fujiwara Y, Ohashi T. Method of purifying single wall carbon nanotubes. Int $\mathrm{J}$ Pharm Pharm Sci 2006; 12:347-83.

31. Allen BL, Kichambare PD, Gou P, Vlasova II, Kapralov AA, Konduru $\mathrm{N}$, et al. Biodegradation of single-walled carbon nanotubes through enzymatic catalysis. Nano Letters 2008;8(11):3899-903.

32. Meher HJ, Kesharwani P, Chaurasia M, Singh A, Chourasia MK. Carbon Nanotubes (CNTs): A Novel Drug Delivery Tool in Brain Tumor Treatment. Elsevier 2018;14:375-96.

33. Ji SI, Byong CB, Young-SL. The effect of carbon nanotubes on drug delivery in an electro-sensitive transdermal drug delivery system. Biomaterials 2010;31:1414-9.

34. Prajapati SK, Jain A, Shrivastava C, Jain KA. Hyaluronic acid conjugated multi-walled carbon nanotubes for colon cancer targeting. Int J Biol Macromol 2019; 123:691-703. 